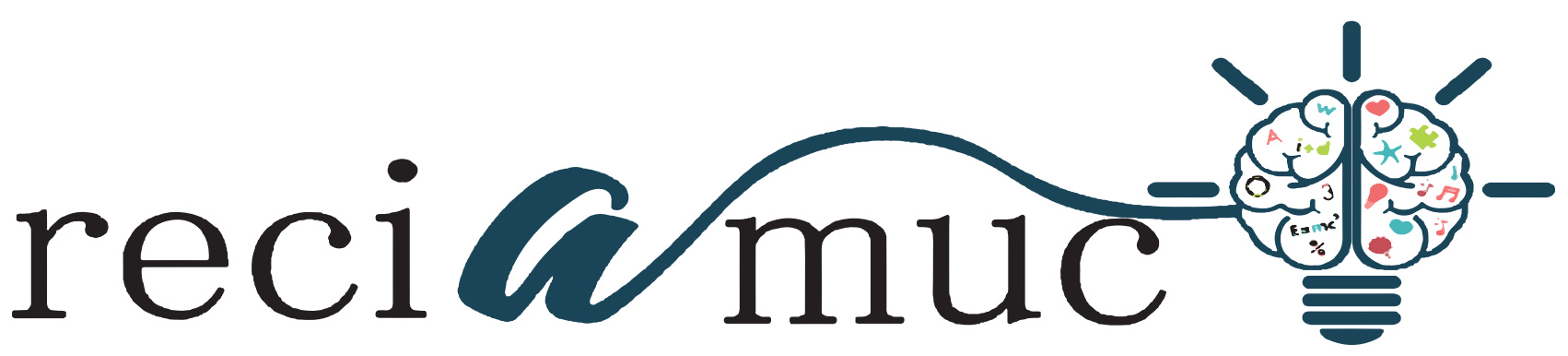

DOI: 10.26820/reciamuc/5.(1).ene.2021.70-77

URL: https://reciamuc.com/index.php/RECIAMUC/article/view/594

EDITORIAL: Saberes del Conocimiento

REVISTA: RECIAMUC

ISSN: 2588-0748

TIPO DE INVESTIGACIÓN: Artículo de Investigación

CÓDIGO UNESCO: 32 Ciencias Médicas

PAGINAS: $70-77$

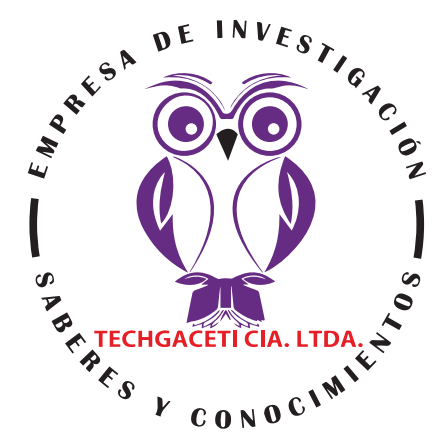

Causas más frecuentes de amenaza de parto prematuro en el Hospital Universitario

Most frequent causes of threatened preterm labor at the University Hospital Causas mais frequentes de ameaça de trabalho de parto prematuro no Hospital Universitário

Jennyffer Maribel Salazar Veloz'; Dana Nicole Guevara Moreira2; Javier Enrique Dominguez Vera³

RECIBIDO: 10/12/2020 ACEPTADO: 29/12/2020 PUBLICADO: 31/01/2021

1. Médico; Investigadora Independiente; Guayaquil, Ecuador; j_saly8711@hotmail.com; (D https://orcid.org/00000003-3183-5839

2. Médico; Investigadora Independiente; Guayaquil, Ecuador; dannanicole_gm@hotmail.com; (D) https://orcid. org/0000-0002-5732-8942

3. Médico; Investigador Independiente; Guayaquil, Ecuador; jd24mas@hotmail.com; $\mathbb{D}$ https://orcid.org/00000002-0512-6951

\title{
CORRESPONDENCIA
}

Jennifer Maribel Salazar Veloz

j_saly8711@hotmail.com

\section{Guayaquil, Ecuador}

〔C RECIAMUC; Editorial Saberes del Conocimiento, 2021 


\section{RESUMEN}

Esta investigación determina los factores de riesgo materno en pacientes con amenaza de parto prematuro atendidas en el Hospital Universitario periodo 2012. Es un estudio descriptivo, retrospectivo, se revisaron 159 historias clínicas que correspondió al universo de estudio. Obteniendo como resultados 302 eventos obstétricos atendidos, 159 fueron amenazas de parto prematuro determinando un 53\%. Las patologías de riesgo de amenaza parto prematuro son: Infecciones del tracto urinario (65\%), infecciones genitales (14\%), oligohidramnios (7\%), ruptura prematura de membranas $(4 \%)$, pielonefritis (4\%) fistula amniótica (3\%), trastornos placentarios (2\%), traumatismo (1\%), bradicardia fetal (1\%); estas patologías pueden diagnosticarse y tratarse en la atención prenatal. Dentro de los antecedentes Gineco-Obstétricos con amenaza de parto prematuro tenemos: primigesta (41\%), nulípara (10\%), secundigestas (25\%); multigestas (24\%).cuya edad gestacional oscila entre 27 - 30 semanas (36\%). La madre con mayor riesgo de amenaza de parto prematuro es de 19-26 años de edad (25\%). Los resultados obtenidos demuestran que la infección de vías urinarias es la patología que con mayor frecuencia se presenta durante el embarazo, con tratamiento adecuado evitaremos complicaciones como es la Amenaza de parto prematuro.

Palabras clave: Parto Prematuro, Amenaza, Causas, Mujeres, Neonato, Gestantes.

\section{ABSTRACT}

This research determines the maternal risk factors in patients with threatened preterm birth attended at the University Hospital in the period 2012. It is a descriptive, retrospective study, 159 medical records were reviewed that corresponded to the universe of study. Obtaining as results 302 obstetric events attended, 159 were threats of premature birth, determining 53\%. The pathologies of risk of threatening premature birth are: urinary tract infections $(65 \%)$, genital infections $(14 \%)$, oligohydramnios (7\%), premature rupture of membranes (4\%), pyelonephritis (4\%) amniotic fistula (3\%), placental disorders (2\%), trauma (1\%), fetal bradycardia (1\%); These pathologies can be diagnosed and treated in prenatal care. Within the Gyneco-Obstetric antecedents with threat of premature birth we have: first pregnant (41\%), nulliparous (10\%), second pregnant (25\%); multigesta (24\%) whose gestational age ranges between 27-30 weeks (36\%). The mother most at risk of threatened preterm birth is $19-26$ years of age (25\%). The results obtained show that urinary tract infection is the pathology that most frequently occurs during pregnancy, with proper treatment we will avoid complications such as the Threat of premature birth.

KeyWords: Premature Delivery, Threat, Causes, Women, Neonate, Pregnant Women.

\section{RESUMO}

Esta investigação determina os factores de risco materno em doentes com ameaça de parto prematuro atendidos no Hospital Universitário no período de 2012. É um estudo descritivo, retrospectivo, foram analisados 159 registos médicos que correspondiam ao universo de estudo. Obtendo como resultados 302 eventos obstétricos assistidos, 159 foram ameaças de nascimento prematuro, determinando 53\%. As patologias de risco de ameaça de parto prematuro são: infecções do tracto urinário (65\%), infecções genitais (14\%), oligoidrâmnios (7\%), ruptura prematura de membranas (4\%), pielonefrite (4\%) fístula amniótica (3\%), distúrbios da placenta (2\%), traumas (1\%), bradicardia fetal (1\%); Estas patologias podem ser diagnosticadas e tratadas em cuidados pré-natais. Dentro dos antecedentes gineco-obstétricos com ameaça de parto prematuro temos: primeira gravidez (41\%), nulípara (10\%), segunda gravidez (25\%); multigesta (24\%) cuja idade gestacional varia entre $27-30$ semanas (36\%). A mãe com maior risco de ameaça de parto prematuro é de 19-26 anos de idade (25\%). Os resultados obtidos mostram que a infecção do tracto urinário é a patologia que mais frequentemente ocorre durante a gravidez, com um tratamento adequado evitaremos complicações como a Ameaça de parto prematuro.

Palavras-chave: Parto Prematuro, Ameaça, Causas, Mulheres, Recém-Nascidos, Mulheres Grávidas. 


\section{Introducción}

Se define como Amenaza de Parto Prematuro al que se produce entre la semana 27 y 37 de gestación, presencia de contracciones uterinas con una frecuencia de 1 cada 10 minutos, de 30 segundos de duración palpatoria, que se mantienen durante un lapso de 60 minutos con borramiento del cérvix uterino del $50 \%$ o menos y una dilatación igual o menor a $3 \mathrm{~cm}$, entre las 22 y 36,6 semanas de gestación. (Cremonte Ortiz, 2007)

En diversas partes del mundo, los partos prematuros continúan representando entre el 5 y el $12 \%$ de todos los nacimientos. (OMS, 2007)

Su frecuencia varía de 5 a 11\% en las regiones desarrolladas y hasta $40 \%$ en algunas regiones muy pobres. En Estados Unidos, en 1981, representó 9,4\% de los embarazos $y$ en 2005; 12,7\%, lo que significa un aumento de más de 30\% en el periodo. (Goldenberg, 2009) (OPS, 2007)

Sin embargo, en algunos grupos de la población se han reportado cifras aún mayores, como en el de adolescentes, en el que la frecuencia alcanza 21,3\%. (Manrique et al. 2013)

La tasa de prematurez en Latinoamérica es de $11 \%$, mientras que en Europa varía entre 5 a $7 \%$. A pesar de los avances en la atención obstétrica, estas cifras no han disminuido en los últimos 40 años. (Iriarte, 2006)

En Ecuador tenemos las siguientes incidencias reportadas. En el hospital Enrique Garcés, en 1984-85: 5,54\%, en 1986: 8,08\%. En el Hospital Gineco-Obstétrico Isidro Ayora, en 1981: 5,45\%, en 1982: 7,93\%, en 1983: 6,06\% en 1993: 8,32\%. La incidencia de prematurez fue de 9,43\% en el 95, lo que significa un ascenso respecto de los años anteriores en la misma institución. (Yánez, 1998)
El propósito de este estudio es establecer la incidencia de Amenaza de parto prematuro e identificar las causas más frecuentes que puede ocasionar un parto prematuro sino se trata a tiempo, la detección temprana de estos factores de riesgo no requiere de tecnología sofisticada y puede realizarse a partir de la primer consulta y desde el primer nivel de atención donde el médico se desempeña, nos permite tomar conductas activas para la prevención, diagnóstico oportuno y tratamiento adecuado de los mismos, de esta forma estaríamos actuando sobre la posibilidad de reducir los partos pretérmino. Se pretende evaluar la incidencia de las causas más frecuente de amenaza de parto prematuro que se presenta en mujeres gestantes en el Hospital Universitario en el periodo 2012.

Proponemos para dicho objetivo emitir charlas educativas sobre las complicaciones que pueden surgir durante el embarazo y las causa que la provocan entre estas las infecciones de vías urinarias, de la misma manera continuar con los controles perinatales mes a mes para así obtener partos a termino

\section{Metodología}

Es un estudio retrospectivo transversal analítico en donde se incluyeron a todas las pacientes que acudieron al servicio de Gineco-Obstetricia del Hospital Universitario con embarazo de 27 a 37 semanas gestación; que cursaban con amenaza de parto prematuro en el periodo 2012. Para realizar este proyecto de investigación nos hemos ayudado mediante revistas médicas, libros, información tecnológica y datos estadísticos basados en historia clínicas del Hospital Universitario.

Previa autorización del Director del Hospital Universitario, se realizó la recolección de datos de las pacientes ingresadas al servicio de Ginecología durante el periodo de estudio La población y muestra se constituye con las pacientes atendidas en el área de Gineco-Obstetricia del Hospital Univer- 
sitario, con embarazo de 27-37 semanas de gestación; se tomaron 302 historias clínicas de pacientes atendidas por diferentes causas. Se ingresaron a hospitalización según riesgo y se utilizaron tocoliticos, antibióticos e inductores de madurez pulmonar;

Se diseñaron instrumentos que permitieron recoger la información necesaria de las historias clínicas de pacientes gestantes con amenaza de parto prematuro atendidas durante el periodo en cuestión.

\section{Resultados}

Durante el periodo de estudio en el Hospital Universitario se atendieron 302 eventos obstétricos, se puede caracterizar que solo 159 muestras eran con diagnóstico definitivo de Amenaza de Parto Prematuro con un $53 \%$, de los cuales 42 pacientes culminaron en parto pretérmino $12 \%$, se excluyeron 143 muestras que están dentro de la 27-37 semana de gestación pero no presentaba diagnóstico de APP y que sin embargo fueron atendidas en el área de Gineco-Obstetricia del Hospital con un $47 \%$.

Estudios realizado tenemos que el Hospital Regional 1 del Instituto Mexicano del Seguro Social, 7.73 \%. Hospital Nacional Hipólito Unanue de Lima, 4,3\%. En Estados Unidos, en 1981, representó $9.4 \%$ de los embarazos $y$, en 2005, 12.7\%. (Villanueva, s/f.)

En nuestro medio tenemos Hospital Enrique Garcés, en 1984-85: 5,54\%, en 1986: 8,08\%. Hospital Gineco Obstétrico Isidro Ayora, en 1981: 5,45\%, en 1982: 7,93\%, en 1983: $6,06 \%$ en 1993: 8,32\% y en el 95 fue 9,43\%. (Yánez, 1998)

La presentación de parto pretérmino fue de 11, 33\% en el Hospital Pablo Arturo Suárez de Quito en el 2006. Sin embargo, se debe destacar la calidad del trabajo obstétrico de nuestro hospital puesto que de un universo de estudio de 159 pacientes respondieron adecuadamente al tratamiento 117 paciente y su embarazo concluyeron con productos a término.
Las patologías asociadas en orden decreciente fueron infecciones del tracto urinario $65 \%$, infecciones del tracto genital 13 $\%$, oligohidramnios7 \%, ruptura prematura de membranas $4 \%$, pielonefritis $4 \%$ fistula amniótica $3 \%$, trastornos placentarios $2 \%$, traumatismo $1 \%$, bradicardia fetal $1 \%$.

\section{Se reportan diferentes incidencias según los distintos estudios así tenemos}

En un estudio de casos y controles realizado en el Instituto Mexicano del Seguro Social de Baja California entre 1997 y 1998, las infecciones cervicovaginal y de vías urinarias estuvieron asociadas al parto pretérmino en 47,9 y $30,2 \%$, respectivamente. El polihidramnios se presenta entre 0,4 y $2,3 \%$ de todos los embarazos; el origen puede ser materno, fetal o idiopático. (Calderón, 2009)

En nuestro medio en el Hospital Gineco Obstétrico Isidro Ayora reportado por Yánez (1998), la ruptura prematura de membranas ocurre en el 30\% de madres de prematuros, siendo la patología más frecuente en estas madres. La segunda patología es el sangrado genital, con una incidencia del $21 \%$. La hipertensión inducida por el embarazo está presente en $21 \%$ y la infección de vías urinarias tiene una incidencia menor $12 \%$.

En Puerto Cabello-Venezuela Faneite y colaboradores (2007) estudiaron 497 amenazas de parto prematuro las cuales tuvieron como antecedente importante infecciones urinarias $64,63 \%$. La 74 mayoría presentaron sintomatología urinaria (poliaquiuria, disuria, escalofrios, fiebre, lumbalgia) 82,31\%.

Iriarte (2009) en su trabajo Parto Pretérmino en pacientes del "Hospital San Juan de Dios"-Oruro 2006 observó como factores predisponentes: 190 (20\%) pacientes presentaron rotura prematura de membrana; 166 (18\%) pacientes presentaron placenta previa; 115 (12\%) presentaron bacteriuria asintomática; 99 (11\%) a polihidramnios; 94 $(10 \%)$ de pacientes con antecedentes de tabaquismo; 82 (9\%) de pacientes con antecedentes de partos pretérmino. 
En lo referente de las 159 pacientes estudiadas el mayor porcentaje (25\%) se encontraban en el rango de 19 a 26 años a la edad, seguido de 27-30 años de edad con un $21 \%$, de $15-18$ años de edad $15 \%$, entre 31-34 años 10\%, mientras que hay menor proporción en las edades avanzadas como 35-38 años de edad 2\%, y por ultimo de 3942 años de edad $2 \%$.

(U) Estos datos coinciden con otros estudios realizados también en nuestro medio así tenemos en el Hospital Gineco-Obstétrico Isidro Ayora las frecuencias más altas de prematurez están en el grupo de madres de $15-19$ años $(20,2 \%)$ y de $20-24$ años $(34,6 \%)$, de tal manera que el $55 \%$ de los recién nacidos son hijos de madres de menos de 24 años. Conforme aumenta la edad materna, la frecuencia de prematurez disminuyó. No se encontró el repunte en madres añosas reportado en otros trabajos (Yánez, 1998)

En Venezuela una investigación reportada por Faneite (2007) en el Hospital Dr. Adolfo Prince Lara, de Puerto Cabello, Estado Carabobo, se estudiaron 294 pacientes en quienes el grupo etario más frecuente fue la edad de 19 años y menos 29,59\%. (Faneite, 2007)

En lo referente a edad gestacional se observa una mayor incidencia de presentación de este síndrome clínico entre las 27 a 30 semanas de gestación con un $36 \%$, seguido por un $35 \%$ entre las 35 y 37 semanas de gestación y entre las 31 a 34 semanas de gestación un $29 \%$.

Se observa un predominio en la presentación de amenaza de parto prematuro en las nulípara con un 10\%, primigestas con un 41 $\%$, secundigestas con un $25 \%$; multigesta dentro de estas tenemos: tercera gesta con un $16 \%$, cuarta gesta $8 \%$ y quinta gesta $0 \%$.

En nuestro medio en el Hospital Gineco-obstétrico Enrique Sotomayor tenemos hallazgos similares así; las pacientes primigestas presentaron una mayor frecuencia el $38 \%$ sobre las pacientes secundigestas y multigestas.

Este no concuerda con evidencias en investigaciones en otros países como lo reportado por Villanueva (2008) en México donde hay predominio en multigrávidas con antecedente de aborto en el primer trimestre y en las multigrávidas con antecedente de parto prematuro o aborto en el segundo trimestre.

La causa más frecuente que provoco amenaza de parto prematuro fue la infección de vías urinarias con un $65 \%$.

En un estudio de casos y controles realizado en el Instituto Mexicano del Seguro Social de Baja California entre 1997 y 1998, las infecciones cervicovaginal y de vías urinarias estuvieron asociadas al parto pretérmino en 47,9 y $30,2 \%$, respectivamente.

En nuestro medio en el Hospital Gineco Obstétrico Isidro Ayora reportado por Yánez, la ruptura prematura de membranas ocurre en el 30\% de madres de prematuros, siendo la patología más frecuente en estas madres.

En Puerto Cabello-Venezuela Faneite y colaboradores (2007) estudiaron 497 amenazas de parto prematuro las cuales tuvieron como antecedente importante infecciones urinarias $64,63 \%$.

En lo referente de las 159 pacientes estudiadas el mayor porcentaje (25\%) se encontraban en el rango de 19 a 26 años a la edad.

Estos datos coinciden con otros estudios realizados también en nuestro medio así tenemos en el Hospital Gineco-Obstétrico Isidro Ayora las frecuencias más altas de prematurez están en el grupo de madres de 15-19 años (20,2\%) y de 20-24 años $(34,6 \%)$, de tal manera que el $55 \%$ de los recién nacidos son hijos de madres de menos de 24 años.

En lo referente a edad gestacional se observa una mayor incidencia de presentación de este síndrome clínico entre las 27 a 30 
semanas de gestación con un $36 \%$.

Se observa un predominio en la presentación de amenaza de parto prematuro en las primigestas con un $41 \%$, En nuestro medio en el Hospital Gineco-obstétrico Enrique Sotomayor tenemos hallazgos similares así; las pacientes primigestas presentaron una mayor frecuencia el 38\% sobre las pacientes secundigestas y multigestas

\section{Conclusión}

El estudio realizado nos permite establecer la incidencia de amenaza de parto prematuro durante el año 2012 en el Hospital Universitario, de los cuales 159 pacientes con diagnóstico de Amenaza de parto prematuro lo que corresponde a un $53 \%$.

La madre con mayor riesgo de amenaza de parto prematuro es una mujer de 19-26 años de edad con un (25\%).

Entre las patologías que elevan el riesgo de parto pretérmino están: Infecciones del tracto urinario (65\%), infecciones del tracto genital (14\%), oligohidramnios (7\%), ruptura prematura de membranas (4\%), pielonefritis (4\%) fistula amniótica (3\%), trastornos placentarios $(2 \%)$, traumatismo $(1 \%)$, bradicardia fetal (1\%). y lo más interesante es que la mayoría de estas patologías pueden diagnosticarse y tratarse en la atención prenatal.

En lo que respecta a los antecedentes gineco-obstétricos podemos concluir que la paciente con amenaza de parto prematuro es fundamentalmente una primigesta (41\%), nulípara (10\%), secundigestas con un (25 $\%)$; multigesta dentro de estas tenemos: tercera gesta con un (16\%), cuarta gesta (8\%) y quinta gesta (0\%).cuya edad gestacional oscila entre 27 - 30 semanas (36\%).

La detección temprana de algunos factores de riesgo aquí identificados no requiere de tecnología sofisticada y puede realizarse a partir de la primera consulta y desde el primer nivel de atención donde el médico general se desempeña.
Por esto es indispensable, además de destinar los recursos tecnológicos y humanos necesarios para atender a los recién nacidos prematuros, que las autoridades sanitarias supervisen el acceso y cabal cumplimiento del control prenatal, y establezcan un sistema de referencia prenatal eficaz en el que todas las mujeres con embarazo de alto riesgo se identifiquen, se traten de forma adecuada en las unidades de atención primaria y se transfieran oportunamente a los hospitales especializados en la atención de estados materno-fetales de alto riesgo y que cuenten con unidades de cuidados intensivos neonatales.

Este resultado determina una señal de alarma para los profesionales de la salud, ya que se debe poner manos a la obra para que en un futuro no muy lejano, en lo posible inmediato, estas cifras disminuyan; cabe aclarar que este objetivo no se limita a un frío cálculo estadístico, sino a la consecuencia indirecta de una menor cantidad de amenaza de partos prematuro.

Se entiende que el primer paso para este cambio, era el análisis de los factores de riesgo, que, aunque algunos son conocidos, quisimos poner en evidencia cuales eran los que presentaban clara relación estadística con esta población en particular.

Detección temprana de estos factores de riesgo no requiere de tecnología sofisticada y puede realizarse a partir de la primer consulta y desde el primer nivel de atención donde el médico se desempeña, nos permite tomar conductas activas para la prevención, diagnóstico oportuno y tratamiento adecuado de los mismos, de esta forma estaríamos actuando sobre la posibilidad de reducir los partos pretérmino.

Ello obliga a retomar los programas preventivos básicos, como son estimular la educación general, avanzar en la orientación sexual y responsabilidad paterna oportuna, y aumentar la cobertura y calidad de un control prenatal precoz. 


\section{Bibliografía}

Althabe Fernando; Carroli, Guillermo; Lede, Roberto; Belizan José M; Althabe, Omar H. (2013) El parto pretérmino: detección de riesgos y tratamientos preventivos.Revista Panamericana de salud pública. (5), 6. Washington. Disponible en la Web: <www.scielosp.org/scielo.php?pi$\mathrm{d}=$ S1020-49891999000500001\&script=sci_arttext>

Atef, Darwish; Ehab, M; Elnshar, Suha M. Hamadeh; Mohammad, H. Makarem. (2012). Relación de la vaginosis bacteriana con el parto y la ruptura de membranas prematuros. (33), 6 :781-787. Disponible en Web <http://www.cochrane.ihcai.org/programa_seguridad_paciente_costa_rica/pdfs/23_ Tratamiento-y-Prevencion-del-Parto-Pretermino. pdf

Calderón, Juvenal, et al. (2009) Factores de Riesgo Materno Asociados al Parto Pretérmino. Disponible en Web <www.imss.gob. mx/NR/rdonlyres/40BDF015-9DB5/Factoresriesgomaterno.pdf 200904 $28>$

Cremonte Ortiz, Alejandra Elizalde. (2007) Amenaza De Parto Pretérmino. Diagnostico. Conducta. Disponible en Web: <http://med.unne.edu.ar/posgrado/cursomedgral/clases/240207ec.pdf>

Faneite, Pedro, et al. (2007) Amenaza de Parto Prematuro e Infección Urinaria. Revista Obstetricia y Ginecología de Venezuela. 66 (1):1-6.

Goldenberg, R. (Enero, 2009) The management of preterm labor. Obstetrics and Gynecology. (25):1020- 1037. Disponible en Web <http://www. geosalud.com/embarazo/embarazopretermino. htm 200904 >
Iriarte, Carlos. (2006) Parto Pretérmino en Pacientes del Hospital San Juan de Dios. Universidad Privada del Valle. Oruro-Bolivia. Disponible en Web $<$ http://www.univalle.edu/publicaciones/revista_salud/revista05/pagina05.htm 20090901 >

Manrique, Riscarle; Rivero, Airlen; Ortunio, Magaly; Rivas, Marianela; Cardozo, Rosa; Guevara, Harold. (2013). Parto Pretérmino En Adolescentes. Revista de Obstetricia y Ginecología de Venezuela. (68), 3. Caracas. Disponible en la Web </www.scielo.org.ve/scielo.php?pi$\mathrm{d}=$ S0048-77322008000300002\&script=sci_arttext>

OMS. (2007) Situación de Salud en las Américas: indicadores básicos. (Comunicado)

OPS. (2007) Ecuador Salud en las Américas: países, (2): 299-316

Villanueva Egan, Luis Alberto; Contreras Gutiérrez, Ada Karina; Pichardo Cuevas, Mauricio; Rosales Lucio, Jaqueline. (2008) Perfil epidemiológico del parto prematuro. México. Disponible en la Web $<\mathrm{h}$ ttp://www. nietoeditores.com.mx/download/gineco/2008/septiembre2008/Ginecol-542-8.pdf>

Yánez, Nery. (Septiembre, 1998) Perfiles Epidemiológicos de la Madre de Recién Nacido Prematuro en el Hospital Gineco-Obstétrico Isidro Ayora de Quito. Revista Ecuatoriana de Pediatría: 12- 16. 


\section{CITAR ESTE ARTICULO:}

Salazar Veloz, J. M., Guevara Moreira, D. N., \& Dominguez Vera, J. E. (2021). Causas más frecuentes de amenaza de parto prematuro en el Hospital Universitario. RECIAMUC, 5(1), 70-77. https://doi.org/10.26820/reciamuc/5.(1). ene.2021.70-77

\section{(i) (2) (2) \\ BY NC SA}

CREATIVE COMMONS RECONOCIMIENTO-NOCOMERCIAL-COMPARTIRIGUAL 4.0. 